\title{
Triply Helical Giant Domain with \\ Homochirality in a Terpolymer Blend System
}

Yuta Miyamori, $\dagger^{11}$ Jiro Suzuki, $\$ \S$ Yoshitaka Aoyama, \# Tomohiro Mihira, \#, Yushu

$$
\text { Matsushita }{ }^{*}{ }^{2)}, \text { Atsushi Takano, }{ }^{*}+
$$

$\dagger$ Department of Molecular and Macromolecular Chemistry, Graduate School of

Engineering, Nagoya University, Furo-cho, Chikusa-ku, Nagoya 464-8603 Japan

†Computing Research Center, High Energy Accelerator Research Organization, 1-1

Oho, Tsukuba, Ibaraki 305-0801, Japan

§Information System Section, J-PARC Center, 2-4 Shirakatashirane, Tokai, Ibaraki

$$
\text { 319-1195, Japan, }
$$

\#JEOL Ltd., 1-2 Musashino, 3-Chome Akishima, Tokyo 196-8558, Japan 


\section{Structures of terpolymer/homopolymer $90 / 10$ blends}

Self-assembled structures of terpolymer/homopolymer blends are compared in Figure

S1; a) $\mathrm{S}_{1} \mathrm{IS}_{2} \mathrm{P}-3 / \mathrm{S}_{\mathrm{h}}=90 / 10$, and b) $\mathrm{S}_{1} \mathrm{IS}_{2} \mathrm{P}-4 / \mathrm{S}_{\mathrm{h}}=90 / 10$. The ordering of helical domains in

Figure $\mathrm{S} 1 \mathrm{a}$ is better than that of neat tetrablock, $\mathrm{S}_{1} \mathrm{IS}_{2} \mathrm{P}-3$ in Figure 1a but not quite good,

while the domain ordering in Figure $\mathrm{S} 1 \mathrm{~b}$ is much better than that of neat tetrablock,

$\mathrm{S}_{1} \mathrm{IS}_{2} \mathrm{P}-4$ in Figure $1 \mathrm{~b}$.
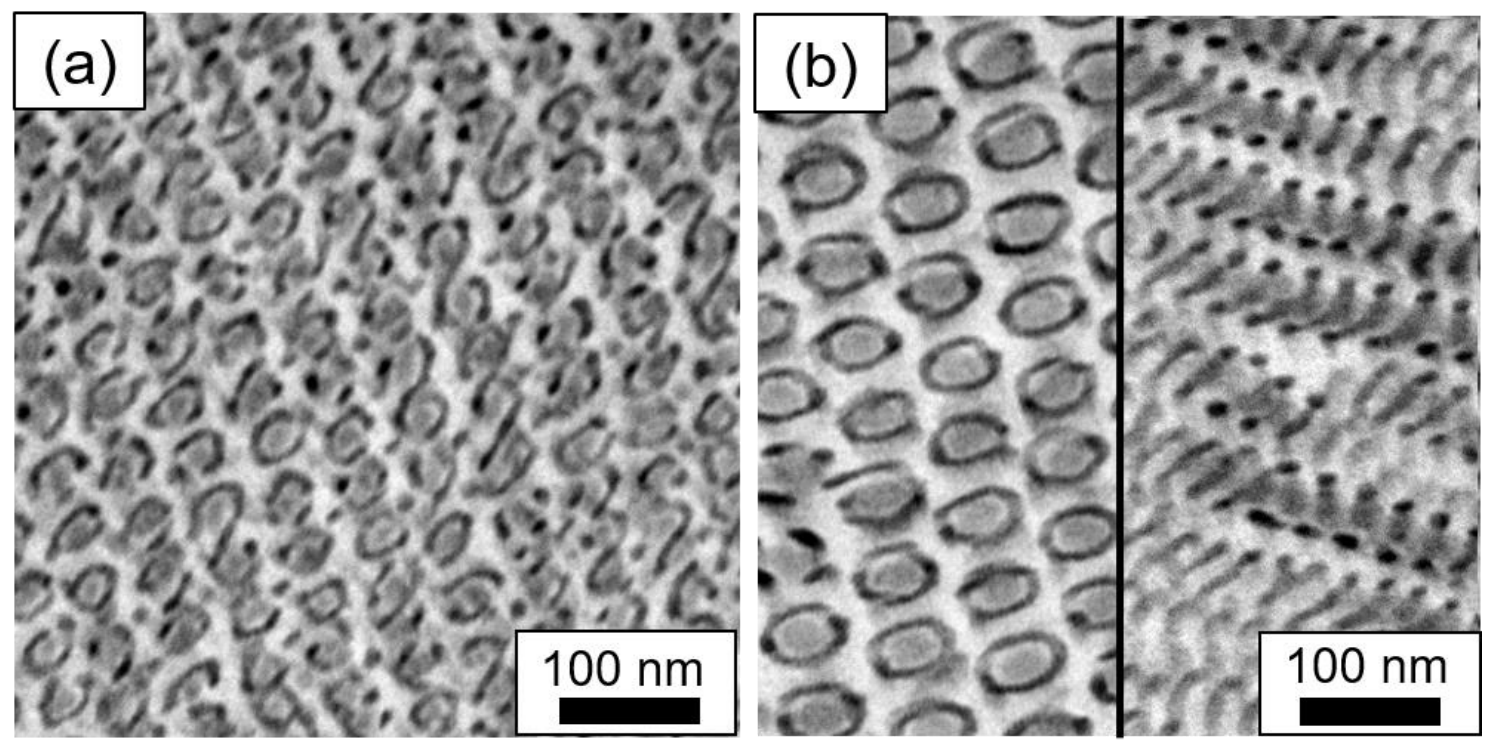

Figure S1 Bright field transmission electron micrographs of two kinds of tetrablock terpolymer/polystyrene homopolymer $\left(\mathrm{S}_{\mathrm{h}}\right)$ blends; a) $\mathrm{S}_{1} \mathrm{IS}_{2} \mathrm{P}-3 / \mathrm{S}_{\mathrm{h}}=90 / 10$ and b) $\mathrm{S}_{1} \mathrm{IS}_{2} \mathrm{P}-$ $4 / \mathrm{S}_{\mathrm{h}}=90 / 10$. Sample specimens were stained with $\mathrm{OsO}_{4}$. 


\section{Detaile d s tructure of $S_{1} I S_{2} P-4 / S_{h}=80 / 20$}

A bright field TEM image of $\mathrm{S}_{1} \mathrm{IS}_{2} \mathrm{P}-4 / \mathrm{S}_{\mathrm{h}}=80 / 20$ is in Figure $\mathrm{S} 2$, wherein inner and outer diameters of each helix are added. The sample specimen was stained with $\mathrm{OsO}_{4}$, and hence helical domain of I phase was mostly highlighted.

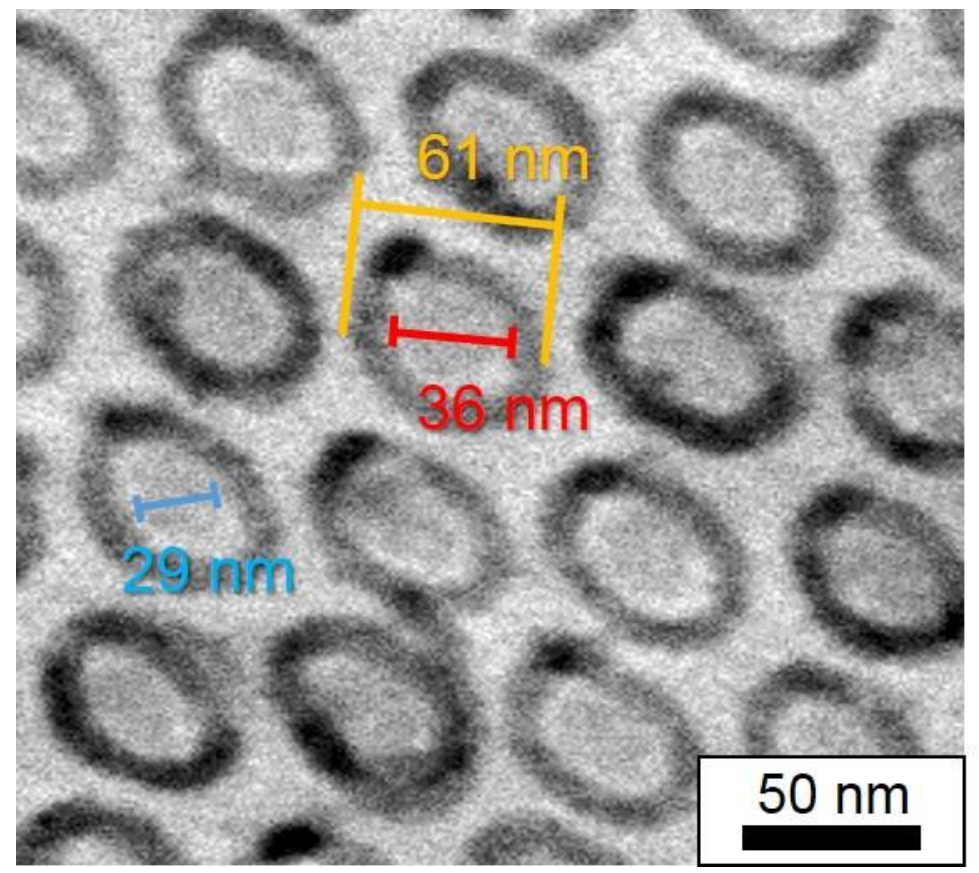

Figure S2 A typical TEM image of a tetrablock terpolymer/polystyrene homopolymer $\left(\mathrm{S}_{\mathrm{h}}\right)$ blend. The blend ratio is $\mathrm{S}_{1} \mathrm{IS}_{2} \mathrm{P}-4 / \mathrm{S}_{\mathrm{h}}=80 / 20$. Sample specimen was stained with $\mathrm{OsO}_{4}$. 


\section{III. r-SAXS information of $S_{1} I S_{2} P-4 / S_{h} 90 / 10$ and $S_{1} I S_{2} P-4 / S_{h} 80 / 20$}

Figure S3a and -S3b are two-dimensional maps of r-SAXS data for $\mathrm{S}_{1} \mathrm{IS}_{2} \mathrm{P}-4 / \mathrm{S}_{\mathrm{h}} 90 / 10$ and $\mathrm{S}_{1} \mathrm{IS}_{2} \mathrm{P}-4 / \mathrm{S}_{\mathrm{h}} 80 / 20$, respectively, both of which are revealing hexagonal patterns of isolated domains, helices in the present case. We can recognize diffraction spots at 10, 11 and 20, however, it is hard to assign the others since higher order peaks are apparently getting vague and become the defused ones. This is because we are observing many grains with their typical size of $10 \mu \mathrm{m}$ whose crystallographic axes are rotating to some extent, the phenomenon can normally be recognized for the regular morphology of hexagonallypacked cylinders in matrix. In Figure S4c, circular averaged intensities are compared, and here the locations of diffraction spots are ca. $3 \%$ smaller in terms of $q(=4 \pi \sin \theta / \lambda)$, where $2 \theta$ and $\lambda$ are scattering angle and wavelength of $\mathrm{X}$-ray, for $\mathrm{S}_{1} \mathrm{IS}_{2} \mathrm{P}-4 / \mathrm{S}_{\mathrm{h}} 80 / 20$ than for $\mathrm{S}_{1} \mathrm{IS}_{2} \mathrm{P}-4 / \mathrm{S}_{\mathrm{h}} 90 / 10$, indicating that lattice parameter of the hexagonal assembly is getting larger with adding homopolymer to terpolymer. 
(a)

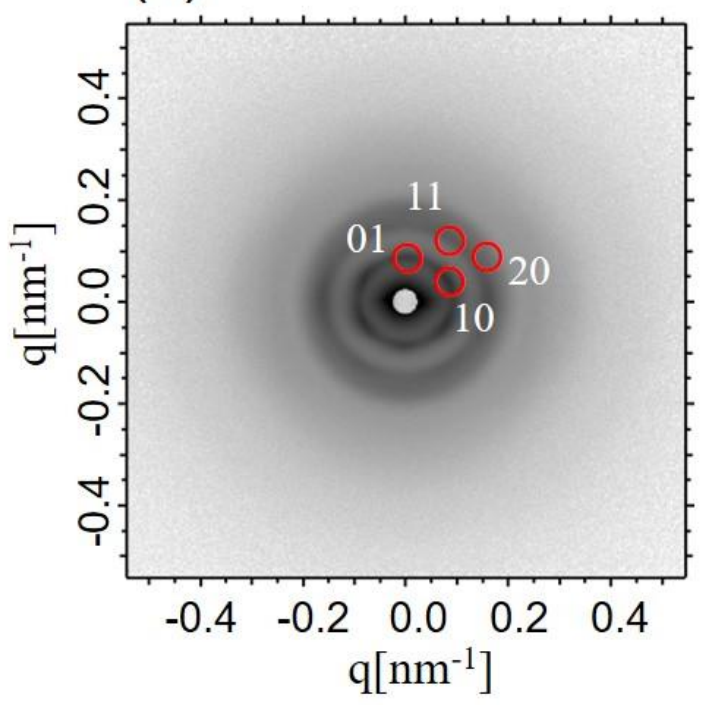

(b)

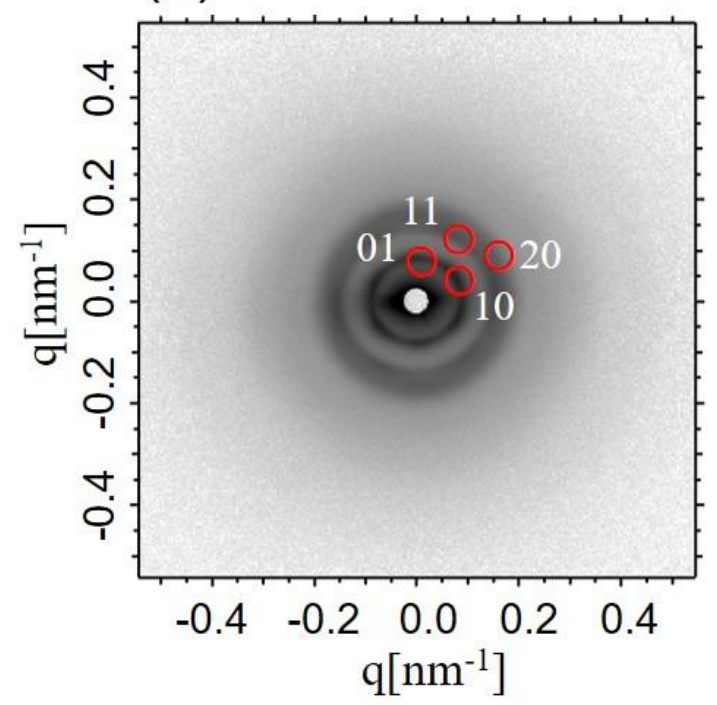

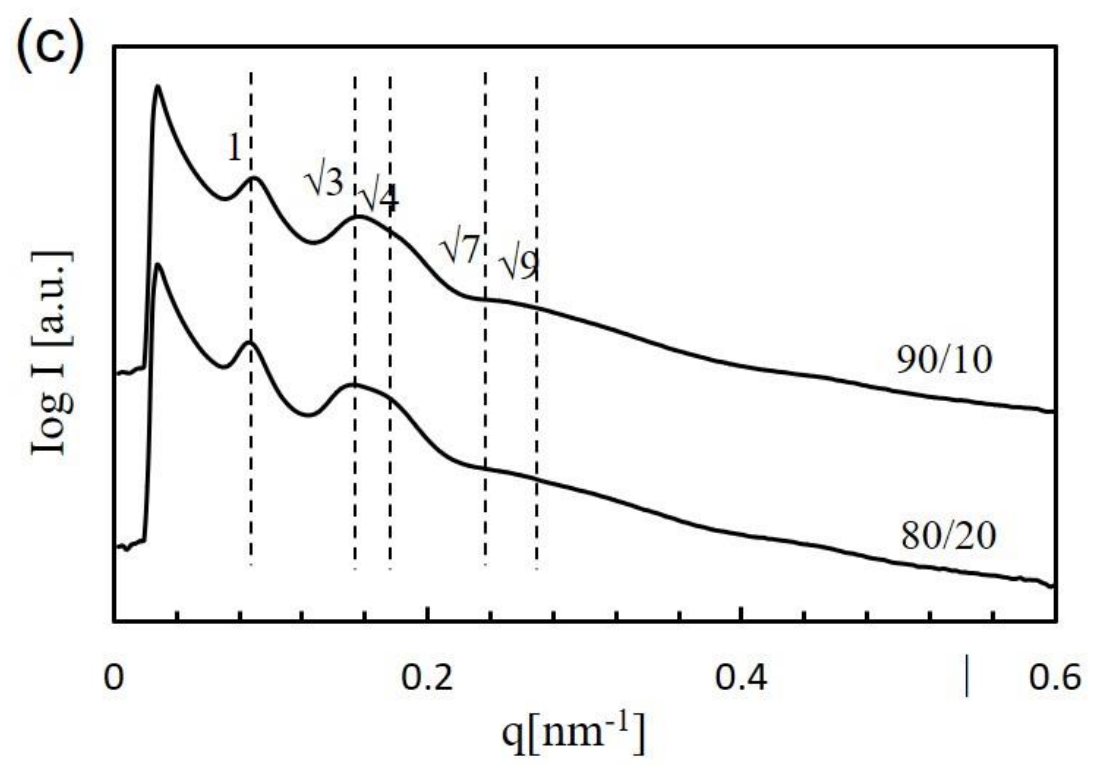

Figure S3 Regular SAXS intensities for block terpolymer/homopolymer blends.; 2Dmaps of a) $\mathrm{S}_{1} \mathrm{IS}_{2} \mathrm{P} / \mathrm{S}_{\mathrm{h}}=90 / 10$ and b) $\mathrm{S}_{1} \mathrm{IS}_{2} \mathrm{P} / \mathrm{S}_{\mathrm{h}}=80 / 20$, and c) their circular averaged data. 


\section{Homochirality and he te rochirality}

Two types of self-assembled manners from helical domain are compared: a) homochirality, in which helical sense is all the same, b) heterochirality whose sense is heterogeneous. Helical senses are schematically shown as colored arrows, green for lefthanded and yellow for right-handed.

a
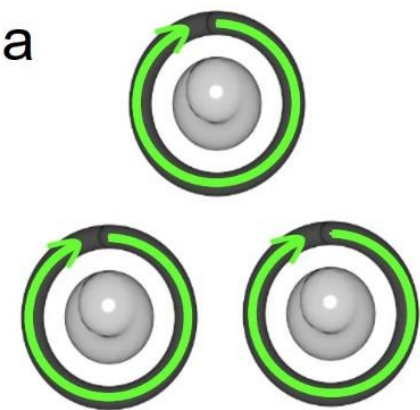

C

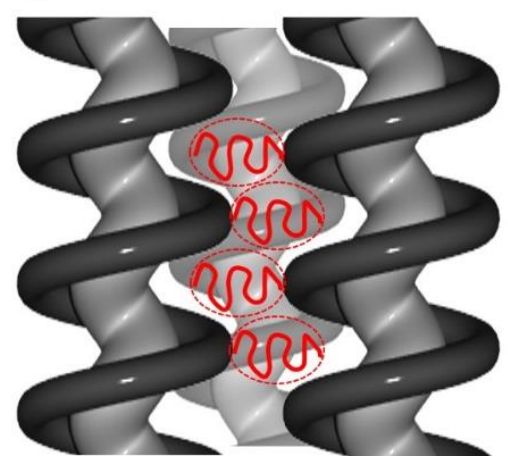

b

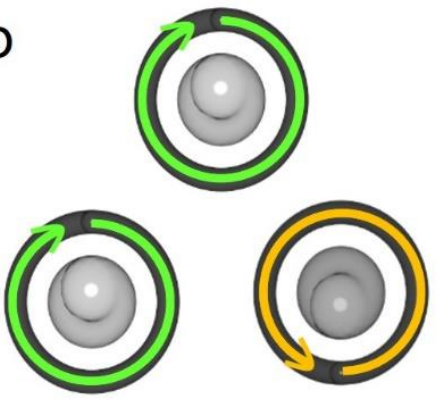

d

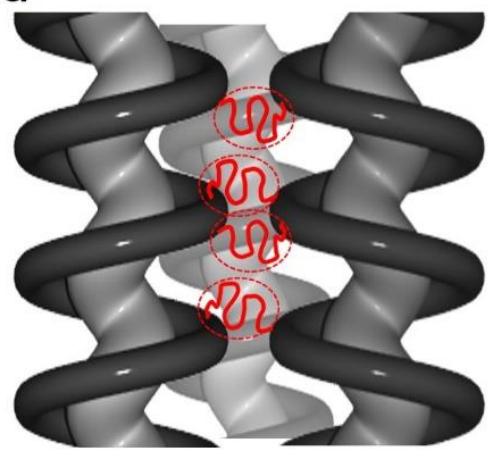

Figure S4 Top views of a) homochiral and b) heterochiral fashions of helices assembly and c) and d) are their side views. 
Here we should compare the packing manners of the outer long $\mathrm{S}_{1}$ chains as matrix phase to understand the priority choice of the homochiral assembly. Figure S4a and S4b are top views of hexagonal packing of helices, while Figure S4c and S4d are their side views together with possible chain conformations. It is evident that elongated $\mathrm{S}_{1}$ chains can fill the open space efficiently in homochiral fashion as shown in Figure S4c, whereas $\mathrm{S}_{1}$ chains must be tightly filled in heterochiral one as evidently compared in Figure S4d, and hence this arrangement in space must be inhibited. The present unusual and noble homochiral structure was probably created mainly because of this simple reason. 


\section{Cylinder-helix transition}

Figure S5a shows 2D-TEM image of $\mathrm{S}_{1} \mathrm{IS}_{2} \mathrm{P}-3 / \mathrm{S}_{\mathrm{h}}=80 / 20$ blend and Figure $\mathrm{S} 5 \mathrm{~b}$ shows 3D-constructed images of the I domains in the same sample. From these image, it is evident that the straight cylindrical domain is transforming into helical cylinders.

(a)

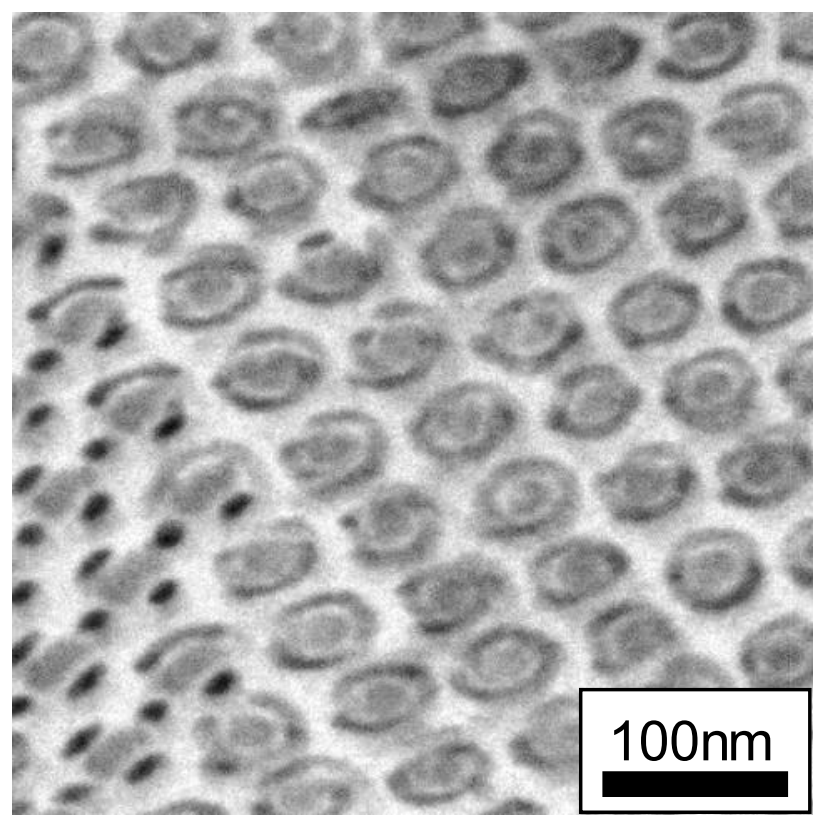

(b)

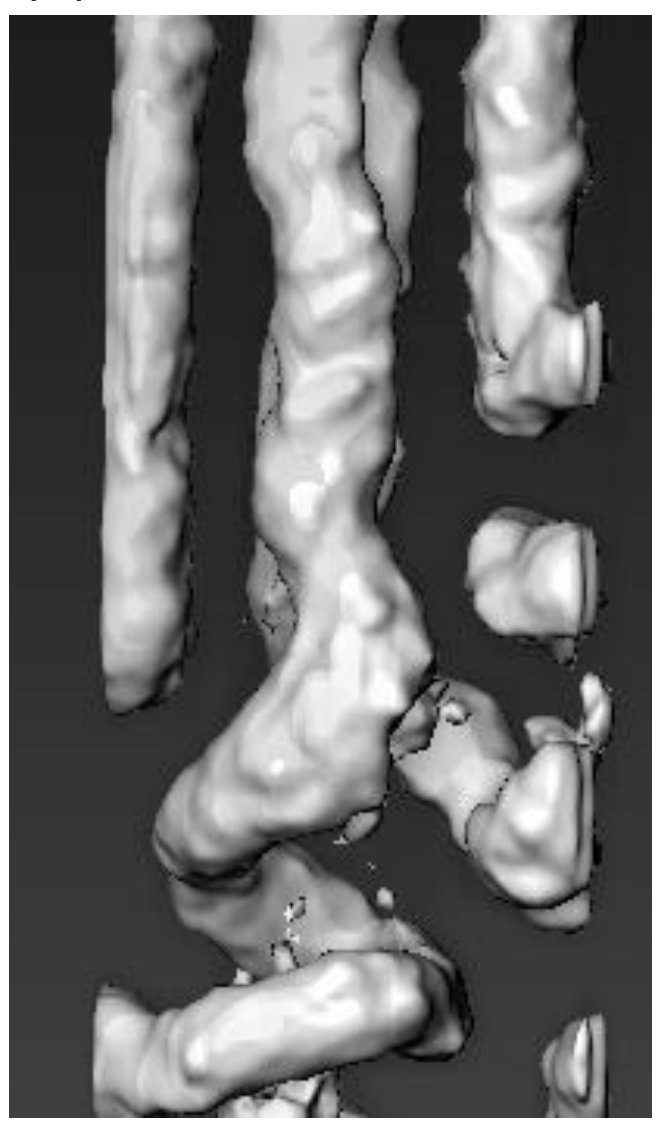

Figure S5 (a) 2D-TEM image of the $\mathrm{S}_{1} \mathrm{IS}_{2} \mathrm{P}-3 / \mathrm{Sh}=80 / 20$ blend and (b) 3D-tomography image representing the morphological transformation in the same sample. 